\title{
CONDICIONES DEL ENTORNO LABORAL INFORMAL Y SUS IMPLICACIONES EN EL RIESGO DE DEPRESIÓN PARA PERSONAS MAYORES EN CIUDAD JUÁREZ, MÉXICO
}

\author{
Diego Sánchez-González \\ Universidad Nacional de Educación a Distancia \\ dsanchezg@geo.uned.es \\ ORCID ID: http://orcid.org/0000-0002-4174-4546 \\ Magdalena Medrano-Ramos \\ Universidad de Guadalajara, México \\ magdalena.medrano@outlook.com \\ ORCID ID: https://orcid.org/0000-0002-1806-258X \\ Vicente Rodríguez-Rodríguez \\ Centro de Ciencias Sociales y Humanas, Consejo Superior de Investigaciones Científicas \\ vicente.rodriguez@cchs.csic.es \\ ORCID ID: http://orcid.org/0000-0002-8812-6841
}

Recibido: 18/10/2018; Aceptado: 25/05/2020; Publicado: 17/06/2021

\begin{abstract}
Cómo citar este artículo/citation: Sánchez-González, Diego; Medrano-Ramos, Magdalena y Rodríguez-Rodríguez, Vicente (2021). Condiciones del entorno laboral informal y sus implicaciones en el riesgo de depresión para personas mayores en Ciudad Juárez, México. Estudios Geográficos, 82 (290), e055. https://doi.org/10.3989/estgeogr.202068.067

RESUMEN: En América Latina muchas personas mayores trabajan en empleos informales; sin embargo, existe un limitado conocimiento sobre cómo sus condiciones laborales (horas de trabajo, ambiente, seguridad) influyen en su salud física y metal, y su calidad de vida. El objetivo de esta investigación es analizar las implicaciones del ambiente laboral en el riesgo de síntomas depresivos en personas mayores con empleos informales en Ciudad Juárez, México. La metodología analiza los datos de una encuesta intencional a trabajadores informales de 65 y más años, utilizando técnicas cuantitativas (intervalos de confianza, correlaciones, chi-cuadrado, Odds Ratio, regresión logística binaria, ANOVA y Post Hoc). Los resultados indican que el riesgo de depresión está asociado con las condiciones socioeconómicas, capacidades funcionales y de salud, hábitos de consumo, y demandas del entorno laboral físico y social. Este riesgo varía según el lugar de trabajo, especialmente en comercios ambulantes y vía pública, donde también está relacionado con problemas de salud, económicos y consumo de alcohol. Se propone revisar los programas de salud pública y empleo para prevenir amenazas para la salud e integridad de personas mayores con empleos informales.
\end{abstract}

PALABRAS CLAVE: envejecimiento, síntomas depresivos, empleo precario, riesgos ambientales, gerontología ambiental, México.

\section{Conditions of the informal work environment and its implications for the risk of depression for the elderly in Ciudad Juarez, Mexico}

ABSTRACT: In Latin America many older people work in informal jobs; however, there is limited knowledge about how their working conditions (work hours, environment, safety) influence their physical and mental health, and their quality of life. This research aims at examining the impact of workplace environment on depressive symptoms among the informally-employed elders in Ciudad Juarez, Mexico. The methodology analyzes the data from an intentional survey of informal workers aged 65 and over, using quantitative techniques (confidence intervals, correlations, chi-square, Odds Ratio, binary logistic regression, ANOVA, and Post Hoc). Results indicate that the risk of depression is associated with people' socioeconomic conditions, functional and health capacities, consumption habits, and with the physical and social working environment demands. This risk varies according to the workplace, especially in commerce and street shops, where it is also related with health, economic and alcohol-consumption problems. A strategy to review public health and employment programs to prevent threats for health and integrity of older people with informal employment is proposed.

KEY WORDS: aging, depressive symptoms, precarious work, environmental risks, environmental gerontology, Mexico. 


\section{INTRODUCCIÓN}

En este siglo XXI se prevé que en las ciudades se incremente la población envejecida en riesgo de padecer enfermedades crónicas, como depresión, constituyéndose en una de las principales causas de sufrimiento que limita la calidad de vida de los adultos mayores (Ávila-Funes et al., 2007; Sánchez-González, 2015). Hoy la depresión es la cuarta causa de incapacidad laboral y afecta al 15\% de la población de 65 y más años. También, se estima que esta enfermedad es la segunda causa de pérdida de años de vida saludables a escala mundial y la primera en países desarrollados (Urbina et al., 2007), lo que tiene importantes repercusiones en materia laboral y gasto de salud pública (Tuesca et al., 2003; Lavretsky et al., 2013).

Los profesionales de la salud suelen percibir los síntomas de la depresión como propios de la vejez (Dunlop et al., 2005; Von-Mühlenbrock et al., 2011). Sin embargo, sus consecuencias son devastadoras sobre la comorbilidad y las capacidades funcionales de las personas mayores (Gallo y Rabins, 1999; AguilarNavarro y Ávila-Funes, 2007; Sarró et al., 2013), suponiendo un riesgo elevado de aislamiento, suicidio (Lyness et al., 1998; Conwell et al., 2012), incapacidad y dependencia (Muñoz et al., 2004). Por ello, la depresión necesita ser estudiada entre la población adulta mayor en relación a una multiplicidad de factores biológicos, psicológicos, sociales y ambientales (Tapia et al., 2000; Van-Gool et al., 2007), como exclusión social, duelo, abandono, violencia, y estrés social y laboral (Martínez et al., 2007; Verhaak et al., 2014). El estrés laboral es uno de los principales factores de riesgo de depresión entre la población activa, siendo un desencadenante del absentismo laboral y del deterioro de la salud (Karasek y Theorell, 1990; Peiró, 1999; Tytherleigh et al., 2007). Así, los estudios previos indican la preocupación social e institucional para enfrentar el estrés laboral en el lugar de trabajo y sus implicaciones en la depresión (Seligman, 2008).

El aumento de la esperanza de vida y el retraso en la edad de la jubilación explican el incremento de la población adulta mayor activa, pero pocas investigaciones se centran en discutir las condiciones laborales y los efectos del trabajo en la población envejecida. Al respecto, se constata que las condiciones laborales determinan su seguridad, y salud física y psicológica (Spector, 1986; García-Machín, 2006).

Distintos estudios defienden que continuar trabajando contribuye positivamente al envejecimiento activo y saludable (Giatti y Barreto, 2003); pero, a la inversa, otras investigaciones advierten que la ocupa- ción laboral de personas mayores se asocia con una disminución del tiempo de ocio y un aumento del riesgo de enfermedades, como la depresión (Geib, 2012). De hecho, los adultos mayores son socialmente vulnerables a los peligros del mercado laboral, sobre todo informal, ya que disponen de menos activos (bajo nivel de estudios, problemas de salud) y estrategias (red de ayuda) para enfrentarlos (Phillips et al., 2008; Millán, 2010). Al respecto, es conocido que envejecer implica un aumento de los problemas de salud, y una pérdida de capacidades funcionales y cognitivas, circunstancia que se ve agravada por la presión que sufre el trabajador en el ambiente laboral informal, sin derechos y prestaciones (pensiones, seguro de salud), así como la exposición a entornos físicos problemáticos, lo que incrementa el riesgo de polifarmacia (probablemente por automedicación) y de consumo de alcohol (Serra y Germán, 2013).

En el sector laboral informal la participación del adulto mayor está condicionada por la discriminación por edad (edadismo) y género en el mercado laboral formal, las presiones sociales y ambientales del lugar de trabajo, y sus capacidades funcionales y cognitivas (Montoya y Montes de Oca, 2009; Ramos, 2017). A su vez, en América Latina los empleos precarios, sobre todo, asociados a actividades económicas informales, suelen implicar mayor riesgo para la salud física y psicológica de los trabajadores, especialmente, cuando se trata de mujeres, inmigrantes, niños y personas adultas mayores (Paz, 2010; Sala, 2013). Dichos riesgos laborales son legitimados y catalogados como residuales por las sociedades modernas, cuyas contradicciones se globalizan, justificándose marcos jurídicos flexibles y gobiernos permisivos con la informalidad en el empleo, la desaparición del estado del bienestar y la creciente desigualdad social (Polanyi, 1989; Bech, 2002).

El trabajo informal engloba aquellas actividades económicas no reguladas por las autoridades laborales en particular y, en general, gubernamentales (Hernández-Laos, 2013). En México las actividades económicas informales se concentran, principalmente, en el sector servicios, y presentan escasos niveles de productividad. Dichas actividades informales son desempeñadas por trabajadores asalariados y autónomos, sobre todo de bajo nivel educativo, que de manera sistemática quedan excluidos de los beneficios y las prestaciones marcadas por la legislación laboral, y son vulnerados sus derechos de acceso a un trabajo digno (contrato de trabajo, salarios mínimos, indemnizaciones por despido), a la seguridad social (pensiones contributivas de jubilación) y a la salud pública 
(seguro de salud amplio). También, los trabajadores informales son más vulnerables al riesgo de exclusión social y abusos laborales, policiales y de autoridades municipales, así como están más expuestos a las inclemencias climáticas, lo que tiene efectos adversos para su salud (Medina-Gómez et al., 2019).

En las últimas cuatro décadas México ha experimentado un progresivo aumento del empleo informal, como mecanismo compensatorio frente a la reducción de los empleos formales, y hoy se estima que 30,8 millones de mexicanos tienen un trabajo informal, lo que representa el 56,9\% de la población activa ocupada (INEGI, 2019). Así, la crisis del estado del bienestar y el temor a la pobreza amparan el auge del empleo informal y la aceptación de la precariedad laboral, que conducen a nuevas formas estructurales de exclusión y marginación (Bauman, 2009). La reciente crisis económica global justificó la reforma en la legislación laboral mexicana en el año 2012, que, en la práctica, está significando un incremento de la precariedad e informalidad laboral, mayor temporalidad y escasa o nula protección social (trabajos sin contrato, sin cotizaciones a la seguridad social, sin acceso a una pensión de jubilación y a seguro de salud) (Quintana y Garza, 2017). Asimismo, la creciente inestabilidad laboral se está traduciendo en mayor desigualdad social y vulnerabilidad a los riesgos de depresión entre la población de bajos ingresos, quienes se ven limitados para acceder a los servicios de salud metal (GarcíaSánchez et al., 2016).

El aumento de la población envejecida en situación de vulnerabilidad social (bajos ingresos, falta de pensión y seguro de salud) explica que el $36,5 \%$ de las personas mayores continúe en activo después de los 65 años (Argüelles, 2014), principalmente, en el trabajo informal y en condiciones de incertidumbre y precariedad laboral (sin derechos laborales, sin prestaciones, bajos salarios, violencia, sobrecarga laboral, siniestralidad) que ponen en riesgo su salud y calidad de vida. Existe, sin embargo, un limitado conocimiento de las externalidades negativas del ambiente laboral informal sobre riesgos para la salud de las personas mayores, como la depresión, así como los riesgos de accidentes laborales. Asimismo, los programas institucionales de empleo para personas mayores (empacadores de supermercado) presentan una escasa evaluación de las posibles externalidades (comorbilidad y depresión) sobre este grupo de edad (Vieco, 2014).

La presente investigación es una aproximación al estudio de las problemáticas condiciones del ambiente laboral y sus implicaciones en el riesgo de depresión para personas mayores ocupadas en empleos informales, a través del análisis de los lugares de trabajo. Al respecto, Ciudad Juárez (México) ha sido seleccionada como caso de estudio, ya que es una de las principales ciudades fronterizas, con casi 1,4 millones de habitantes y con una alta tasa de ocupación informal (33\%) (INEGI, 2015). En dicha urbe residen más de 102.000 personas de 60 y más años, lo que supone el $7,3 \%$ del total, y se estima que un alto porcentaje de este grupo social podría estar ocupado como trabajador informal.

El objetivo es analizar el riesgo de síntomas depresivos en personas mayores con empleos informales por factores ambientales en Ciudad Juárez, México. Se propone contribuir a la discusión científica, así como a la mejora de la gestión de la salud pública y la planificación de los programas de empleo dirigidos a este grupo de edad a nivel local en el contexto de ciudades latinoamericanas. Para ello, se plantearon dos interrogantes: 1) ¿Qué factores están asociados al riesgo de sintomatología depresiva en personas mayores con empleos informales en la urbe?; 2) ¿En qué lugares de trabajo los adultos mayores con empleos informales presentan más riesgo de síntomas depresivos? Además, se formuló una hipótesis de partida $(\mathrm{H} 1)$ : el riesgo de síntomas depresivos en personas mayores con empleos informales está asociado con condiciones socioeconómicas, capacidades funcionales, hábitos de consumo, y demandas del ambiente laboral.

\section{Metodología}

El estudio es descriptivo transversal de los datos de una encuesta no probabilística a personas de 65 y más años con empleos informales y residentes en Ciudad Juárez, México. Para encontrar relación entre el riesgo de síntomas depresivos y el entorno laboral informal de las personas mayores se utilizó un cuestionario que, validado a través de pretest, contenía preguntas relativas a las siguientes dimensiones y sus respectivas variables (Tabla $\mathbf{1}$ ): condición socioeconómica (sexo, edad, estado civil, convivencia, educación, pensión, ingresos, dificultad económica, y seguro de salud), capacidad funcional (estado de salud y discapacidad), hábitos de consumo (polifarmacia y alcohol), empleo (tipología de empleo, lugar de trabajo, antigüedad laboral, deseo de cambiar de trabajo, y accidentalidad laboral), presiones del ambiente físicolaboral (barreras arquitectónicas, iluminación, condiciones climáticas, contaminación, tráfico y distancia), y presiones del ambiente socio-laboral (falta de apoyo, violencia, malas relaciones sociales, discrimina- 
ción, sobrecarga e inseguridad laboral). Cada variable identificada fue objeto de operacionalización, lo que implicó su definición conceptual y procedimental, así como el establecimiento de sus indicadores, unidades de medida, escala y valores.

Dicha encuesta incluía, también, 30 preguntas específicas para evaluar la Escala de Depresión Geriátrica de Yesavage (GDS), empleada para la detección de síntomas de depresión en personas mayores (con y sin alteración cognoscitiva), y como medida de la evolución de esos síntomas (Martínez de la Iglesia et al., 2002; Aguilar-Navarro et al., 2007). Se consideró como riesgo de sintomatología depresiva a todas aquellas personas que dieran resultado positivo ( $\geq 11$ puntos y hasta un máximo de 30 puntos) en la escala GDS (Cabañero et al., 2007).

El tamaño de la muestra estuvo compuesto por 62 personas de 65 y más años con empleos informales según sexo y edad. El trabajo de campo se desarrolló en el mes de septiembre de 2015 en Ciudad Juárez, donde los participantes fueron reclutados aleatoriamente en sus lugares de trabajo, localizados en establecimientos comerciales, pequeños comercios y talleres, y comercios ambulantes y vía pública. Los participantes fueron informados debidamente sobre los fines y métodos de la investigación, así como los beneficios previstos y su derecho de abstenerse, logrando su participación mediante el consentimiento informado. Igualmente, se obtuvo la aprobación del proyecto de investigación por parte del Instituto de Ciencias Biomédicas de la Universidad Autónoma de Ciudad Juárez. El limitado tamaño de la muestra estuvo condicionado por la desconfianza inicial de las personas mayores, lo que está relacionado con la alta inseguridad que vive Ciudad Juárez, considerada una de las urbes más violentas del mundo (CCSPJP, 2014). También, las limitaciones del trabajo de campo impidieron el acceso a información retrospectiva, como el historial familiar y de enfermedad mental de los entrevistados. A pesar del reducido tamaño de la muestra, los datos obtenidos han posibilitado una valiosa aproximación a los efectos del ambiente laboral informal sobre los riesgos para la salud de las personas mayores, como la depresión, así como han estimulado la discusión y la formulación de nuevas hipótesis de trabajo sobre un tema escasamente abordado.

El análisis estadístico bivariado (intervalos de confianza, correlaciones, chi cuadrado) y multivariado (regresión logística binaria) de los datos se desarrolló mediante el uso del sofware SPSS. Se realizaron correlaciones para determinar el nivel de asociación entre el grado de sintomatología depresiva y los factores ambientales. También, se estimó el riesgo de síntomas depresivos en relación a otras variables a partir de Odds Ratio con su respectivo intervalo de confianza al 95\%. Asimismo, se aplicó el modelo de regresión logística binaria para determinar los factores de riesgo de depresión, así como calcular la probabilidad de sintomatología depresiva por estresores ambientales. Además, se determinó la ANOVA de un factor para comprobar si los síntomas depresivos varían según lugar de trabajo, así como el análisis Post Hoc para identificar qué lugar difiere del resto según riesgo. Aquí, cabe reseñar que los análisis ANOVA y Post Hoc se realizaron a partir de la matriz de datos desagregada en tres submuestras o lugares de trabajo: centros comerciales, pequeños comercios y talleres, y comercios ambulantes y vía pública.

\section{RESULTADOS}

\section{Análisis estadístico descriptivo}

El $38,7 \%$ de la muestra eran mujeres y el $61,3 \%$ varones, reflejando no solo la tradicional mayor presencia relativa masculina en el mercado laboral, sino la progresiva incorporación femenina a este sector informal (Tabla 1). La edad mínima fue de 65 años y 80 años la máxima, siendo la edad media 72 años. El estado civil predominante de los participantes era casado (50\%), seguido de viudo $(25,8 \%)$, y soltero $(14,5 \%)$, siendo minoría los separados y en unión libre. Conforme a las formas de convivencia de los participantes, una de cada dos personas vivía con el cónyuge y los hijos, y el $19,4 \%$ solo con los hijos, mientras que el $29 \%$ vivía solo. Asimismo, se evidenciaron diferencias en la convivencia según género, ya que, entre los varones, existía una mayor presencia relativa de aquellos que vivían con el cónyuge, mientras que entre las mujeres predominaban las que convivían con sus hijos y aqueIlas que vivían solas $(\mathrm{OR}=27,12 ; \mathrm{IC} 95 \%=3,27-225,21)$.

El nivel educativo de los participantes reflejó una baja formación, con una media de escolaridad de 4,6 años, así como que el $54,8 \%$ de la muestra no había podido concluir los estudios de primaria, y solo el $4,8 \%$ tenía estudios universitarios. En la misma línea, las personas sin estudios registraban mayor riesgo de no tener derecho a una pensión contributiva $(\mathrm{OR}=5,00$; IC95\%=1,69-14,80) y de mayores dificultades económicas $(O R=8,14 ;$ IC95\%=2,58-25,69). Algo más de la mitad de la muestra, el $56,5 \%$, no contaba con una pensión contributiva de jubilación, una tasa inferior a la media nacional (75\%) (OCDE, 2016), y que afectaba, sobre todo, a mujeres y personas de avanzada edad. Por su 
parte, el 43,8\% percibía una pensión no contributiva de 580 pesos mensuales (32,2 euros), que le permitía cubrir solo el $14,9 \%$ del valor de la canasta básica, situada en los 3.890 pesos (INEGI, 2015). En general, más de ocho de cada diez de los participantes admitía tener dificultades económicas para cubrir sus necesidades básicas (alimentación, salud, transporte y vivienda), sobre todo, mujeres de 75 y más años sin estudios.

El $60 \%$ de los encuestados percibía su salud como regular o mala, y el resto como buena y muy buena. Las mujeres (OR=9,62; IC95\%=2,44-37,89) y las personas de 75 y más años $(O R=6,00$; IC95\%=1,10-32,76) presentaban mayor riesgo de problemas de salud. Uno de cada cuatro participantes tenía algún tipo de discapacidad, principalmente, visual $(14,5 \%)$, motora $(11,3 \%)$ y auditiva (4,8\%). Así, el 41,9\% tenían seguro de salud del ISSSTE e IMSS, el 37,1\% contaban con un seguro de salud limitado en prestaciones (Seguro Popular), el 12,9\% no contaban con ninguno, y un $8,1 \%$ otros seguros. Casi la mitad de los participantes $(48,4 \%)$ consumía de forma habitual tres y más medicamentos (polifarmacia), siendo personas con mayor riesgo de no tener seguro de salud $(O R=9,43$; IC95\%=1,08-82,11), vivir solo (OR=4,13; IC95\%=1,25-13,66), discapacidad (OR=4,67; IC95\%=1,30-16,74), y consumo de alcohol (OR=27,12; IC95\%=3,27-225,21). De hecho, el 24,2\% afirmaba consumir de forma habitual alcohol.

En el análisis del lugar de trabajo, uno de cada dos empleos informales se registró en los centros comerciales, seguido de comercios ambulantes y trabajos en la vía pública $(25,8 \%)$, así como pequeños comercios y talleres (24,2\%). Entre la amplia variedad de trabajos informales destaca el empacador en supermercado (50\%), el vendedor ambulante $(27,4 \%)$, el pepenador (chatarrero, cartonero) $(6,5 \%)$, el camarero $(3,2 \%)$, el limpiabotas $(3,2 \%)$, y con porcentajes inferiores al $2 \%$, albañiles, relojeros, costureros, músicos callejeros, vigilantes y aparcacoches. La antigüedad laboral mínima fue menor a un año y la máxima de 60 años, con una media de 12,5 años. Al respecto, el $45,2 \%$ de los trabajadores informales tenía 10 y más años de antigüedad laboral en su empleo actual, sin embargo, el $21 \%$ tenía menos de 2 años. Asimismo, en relación a la categoría laboral, el 67,7\% de los participantes eran asalariados y el $32,3 \%$ trabajaba por cuenta propia e independiente. La gran mayoría, el 88,7\%, percibía menos de dos salarios mínimos, frente al 3,2\% que ingresaba cuatro salarios mínimos. También, se evidenció que los participantes trabajaban 6 horas al día de media, pero uno de cada cinco $(22,6 \%)$ dedicaba más de 8 horas.
Los problemas del ambiente socio-laboral afectaron a nueve de cada diez participantes, sobre todo, bajos salarios $(90,3 \%)$, violencia o abuso $(51,6 \%)$, sobrecarga laboral $(40,3 \%)$, malas relaciones sociales $(33,9 \%)$ e inseguridad laboral (32,3\%). El mayor riesgo se detectó entre los trabajadores informales de comercios ambulantes y de la vía pública, vendedores ambulantes, pepenadores y limpiabotas (OR=17,89; IC95\%=3,62$88,41)$, así como de los pequeños comercios y talleres $(O R=16,73$; IC95\%=1,70-164,84). Los problemas del ambiente físico-laboral fueron identificados por uno de cada cinco participantes, donde sobresalieron las barreras arquitectónicas $(9,7 \%)$, tráfico y riesgo de atropellos $(6,5 \%)$, contaminación acústica, deficiente iluminación y distancia $(4,8 \%)$. Su mayor riesgo se identificó entre los trabajadores informales de comercios ambulantes y de la vía pública $(\mathrm{OR}=48,4$; IC95\%=8,26-283,56). A la inversa, en los centros comerciales se registraron menos problemas en el ambiente laboral y menos accidentes.

En los últimos seis meses el $12,9 \%$ de los trabajadores informales había sufrido un accidente durante su jornada laboral, una tercera parte de los cuales había sufrido dos o más accidentes. Los participantes con mayor riesgo de sufrir accidentes laborales eran personas sin escolarizar $(O R=20,83$; IC95\%=3,60$120,69)$, con discapacidad física y visual (OR=7,50; IC95\%=1,29-43,45), que consumían alcohol (OR=7,33; IC95\%=1,50-35,87), que trabajaban en comercios ambulantes y vía pública $(O R=6,51 ; I C 95 \%=1,35-31,54)$, sin seguro de salud $(O R=5,88 ; I C 95 \%=1,07-32,24)$, o que vivían solas $(O R=5,26$; IC95\%=1,10-25,05). El riesgo de accidentes laborales se incrementa en lugares de trabajo con altas presiones ambientales (físicas y sociales) en relación con la progresiva disminución de capacidades funcionales de las personas mayores (perdida de salud, discapacidad), así como la ausencia de derechos y coberturas, agravados por las limitadas inspecciones laborales, que no contribuyen a evaluar y registrar de forma fidedigna la accidentalidad y siniestralidad laboral.

Las difíciles condiciones laborales de los trabajadores informales explica que el $38,7 \%$ de los participantes desearía cambiar de lugar de trabajo, sobre todo, aquellos que han sufrido un accidente laboral en los 6 últimos meses (OR=6,00; IC95\%=1,10-32,76), que laboraban en la vía pública, como pepenadores y limpiabotas (OR=4,80; IC95\%=1,10-20,92), y que presentaban mala salud (OR=6,00; IC95\%=1,10-32,76), discapacidad física (OR=12,33; IC95\%=1,38-110,27), polifarmacia $(O R=6,50 ; I C 95 \%=2,06-20,52)$, consumo 
de alcohol $(O R=21,27 ; \mathrm{IC} 95 \%=4,15-109,08)$, o dificultad económica $(\mathrm{OR}=3,33$; IC95\%=1,08-10,24).

En el análisis de la Escala de Depresión Geriátrica de Yesavaje (GDS) se obtuvo una prevalencia de sintomatología depresiva del $41,9 \%$ en grado moderado y severo (Tabla 1 ). Al respecto, el riesgo de síntomas depresivos se asoció con mujeres $(\mathrm{OR}=3,03$; IC95\%=1,05-8,77), personas sin estudios de primaria, que percibían su salud como regular y mala $(\mathrm{OR}=7,70$; IC95\%=2,20-27,72), con discapacidad física y auditiva (OR=6,81;IC95\%=1,30-35,79), polifarmacia (OR=3,34; IC95\%=1,16-9,61), consumo de alcohol (OR=1,84; IC95\%=1,58-5,95), cobertura limitada del servicio de salud (Seguro Popular) o sin seguro de salud (OR=5,43; IC95\%=1,79-16,46), sin pensión (OR=3,39; IC95\%=1,14$10,07)$ y con dificultad económica (OR=4,17; IC95\%=1,35-12,83). Ese riesgo afecta a quienes trabajaban en comercios ambulantes y vía pública $(O R=2,19$; IC95\%=1,69-6,96), como vendedores ambulantes, pepenadores y limpiabotas, donde detectaban problemas del ambiente físico-laboral (deficiente iluminación, distancia y condiciones climáticas extremas) y ambiente socio-laboral (discriminación, malas relaciones sociales y sobrecarga laboral) $(O R=2,00$; IC95\%=1,24-5,63). Por otro lado, la ausencia de síntomas depresivos se asoció con varones menores de 75 años, con buena salud, pensión, seguro de salud, autónomos, y vendedores en pequeños comercios y relojeros en talleres.

A partir de los resultados, se comprobó, primero, la existencia de correlaciones significativas entre la prevalencia de sintomatología depresiva y las variables edad $\left(0,350^{* *}\right)$ y nivel educativo $\left(-0,297^{*}\right)$ (Tabla 1$)$, lo que explica un mayor riesgo de síntomas depresivos entre las personas de 75 y más años con bajo nivel de estudios. En segundo lugar se registraron correlaciones significativas con las variables percepción de la salud $\left(-0,577^{* *}\right)$, polifarmacia $\left(0,532^{* *}\right)$, consumo de alcohol $\left(0,357^{* *}\right)$, discapacidad física $\left(0,317^{*}\right)$ y auditiva $\left(0,253^{*}\right)$, de lo que se desprende la asociación entre el riesgo de síntomas depresivos y los problemas de salud, agravados por el consumo excesivo de medicamentos y alcohol. En tercer lugar, se evidenciaron correlaciones significativas entre la sintomatología depresiva y variables relacionadas con los problemas del ambiente socio-laboral $\left(0,396^{* *}\right)$, como inseguridad laboral $\left(0,323^{*}\right)$, discriminación $\left(0,309^{*}\right)$, sobrecarga laboral $\left(0,304^{*}\right)$, malas relaciones sociales $\left(0,301^{*}\right)$ y abuso $\left(0,261^{*}\right)$. Por último, son también interesantes de destacar las correlaciones entre los síntomas depresivos y variables vinculadas al ambiente físico-laboral $\left(0,305^{*}\right)$, como condiciones climáticas extremas $\left(0,354^{* *}\right)$, deficiente iluminación $\left(0,265^{*}\right)$ y distancia $\left(0,253^{*}\right)$. De lo anterior, se deduce la influencia negativa de un entorno laboral no adecuado sobre la prevalencia de sintomatología depresiva en personas mayores con empleos informales.

Igualmente, la prueba de chi-cuadrado de Pearson evidenció asociación significativa entre los síntomas depresivos y algunas variables categóricas destacadas, como la presencia de mujeres $(0,038)$, trabajar en un pequeño comercio y taller $(0,023)$, trabajar en comercio ambulante y vía pública $(0,041)$, y accidente laboral $(0,024)$. Todos ellos son grupos vulnerables, como mujeres con empleos informales en pequeños comercios, comercios ambulantes y vía pública, así como con riesgo de accidentes laborales. En definitiva, los resultados confirmaron la hipótesis de partida, al evidenciarse que el riesgo de síntomas depresivos en personas mayores con empleos informales está asociado con condiciones socioeconómicas, capacidades funcionales, hábitos de consumo, y demandas del ambiente laboral.

\section{Análisis estadístico multivariado}

La técnica predictiva de la regresión logística binaria se empleó para establecer los factores que determinan en mayor medida el riesgo de síntomas depresivos en personas mayores con empleos informales (Tabla 2). Se constata que existe una alta probabilidad $(75,8 \%)$ de predicción de la sintomatología depresiva por la salud (mala salud percibida) y los estudios (sin estudios). También, ha sido posible comprobar que existe una alta probabilidad $(83,4 \%)$ de predicción de los síntomas depresivos por los estresores del ambiente físico-laboral (distancias) y socio-laboral (inseguridad laboral) (Tabla 3). Esto significa que el ambiente laboral informal (recorrer largas distancias), vinculado a empleos precarios (pepenador, limpiabotas, vendedor ambulante), tiene repercusiones negativas sobre la salud y las capacidades de adaptación, lo que incrementa el riesgo de sintomatología depresiva entre los trabajadores informales de 65 y más años.

El análisis de la ANOVA de un factor permitió comprobar que los síntomas depresivos variaban de manera significativa según lugar de trabajo $(0,031)$ (Tabla 4). También, a través del análisis Post Hoc de la varianza, mediante el test de Bonferroni, se identificó que los comercios ambulantes y vía pública presentan mayores diferencias significativas, en relación al resto de lugares de trabajo, como pequeños comercios y talleres $(0,028)$, y centros comerciales $(0,045)$ (Tabla 5$)$. Igualmente, con objeto de identificar los factores que determinaban la sintomatología depresiva según lugar de trabajo, la matriz de datos fue desagregada en 
tres submuestras (Tabla 6). Como resultado del análisis de la primera submuestra, la variable personas con síntomas depresivos en centros comerciales se asoció significativamente con las variables salud $(-0,559 * *)$ y polifarmacia $\left(0,516^{* *}\right)$, y, en menor medida, con inseguridad laboral y deseo de cambiar de trabajo. Seguidamente, en la segunda submuestra la variable personas con síntomas depresivos en pequeños comercios y talleres se relacionó de manera significativa con las variables inseguridad laboral $\left(0,784^{* *}\right)$, discapacidad física $\left(0,728^{* *}\right)$, salud $\left(-0,701^{* *}\right)$, malas relaciones sociales $\left(0,681^{* *}\right)$, violencia y sobrecarga laboral $\left(0,650^{* *}\right)$, y en menor medida, nivel educativo y dificultad económica. Asimismo, en la tercera submuestra la variable personas con síntomas depresivos en comercios ambulantes y vía pública se asoció significativamente con las variables malas relaciones sociales $\left(0,733^{* *}\right)$, dificultades económicas $\left(0,663^{* *}\right)$, salud $\left(-0,637^{* *}\right)$, consumo de alcohol $\left(0,618^{* *}\right)$, y polifarmacia $\left(0,600^{* *}\right)$, y en menor medida, violencia y deseo de cambiar de trabajo. De los resultados se deduce que los síntomas depresivos se asocian con la salud en los lugares de trabajo. La sintomatología depresiva, sobre todo en los comercios ambulantes y vía pública, se relaciona con problemas socioeconómicos y hábitos de consumo nocivos (polifarmacia y alcohol). Así, la sintomatología depresiva se asocia con los problemas del ambiente socio-laboral, como violencia, en los pequeños comercios y comercios ambulantes, pero no se encontró asociación en los centros comerciales. Conjuntamente, no se identificaron asociaciones entre los síntomas depresivos y los problemas del ambiente físico-laboral de los diferentes lugares de trabajo.

\section{DisCUSIÓN Y CONCLUSIONES}

La literatura indica la importancia de la depresión en la salud de las personas mayores (Heikkinen y Kauppinen, 2004; Aguilar-Navarro y Ávila-Funes, 2007) y su incremento se asocia, entre otros factores, a cuestiones genéticas y ambientales (Lupien et al., 2009). Sin embargo, pocas investigaciones han examinado empíricamente el estrés laboral asociado a la depresión de personas mayores en activo (Melguizo et al., 2012). Precisamente, existe un escaso conocimiento de los efectos del ambiente laboral informal sobre los síntomas depresivos y, en general, la salud de los trabajadores de avanzada edad en ciudades de América Latina.

Los resultados indican que el riesgo de sintomatología depresiva entre trabajadores informales de 65 y más años se asocia con las condiciones socioeconó- micas (edad, estudios, ingresos, seguro médico), capacidades funcionales (salud y discapacidad), hábitos de consumo (polifarmacia, alcohol), y demandas del ambiente físico-laboral (condiciones climáticas, iluminación, distancia), y del socio-laboral (inseguridad, discriminación, sobrecarga laboral). Esto es especialmente preocupante entre trabajadores informales de avanzada edad y en situación de alta vulnerabilidad social (pobreza, desprotección), de bajos ingresos y de menor apoyo familiar (Tuesca et al., 2003). Al respecto, el limitado acceso a la educación explica la elevada tasa de trabajadores de avanzada edad con bajos ingresos, sin pensión o seguro de salud, así como la necesidad de permanecer en el mercado laboral informal, cuyas precarias condiciones de trabajo, incrementan los riesgos para su salud (Paz, 2010; Escobar, 2012; Álvarez et al., 2016).

El predominio del sexo masculino en este mercado está relacionado con su papel social tradicional de proveedores económicos del hogar, lo que explica la preeminencia de ocupados casados sobre los demás estados civiles (Dechent, 2008). A pesar de ello, las mujeres trabajadoras de avanzada edad y viudas, aunque menos en número, presentan una mayor exposición al riesgo de síntomas depresivos, una problemática social que se puede incrementar por la progresiva incorporación de éstas al mercado laboral informal en la región. También, la experiencia espacial de vivir en soledad, sobre todo, de ellas, se asocia con la depresión y problemas de exclusión social (Sánchez-González, 2009).

El riesgo de sintomatología depresiva es mayor entre trabajadores informales de avanzada edad, con problemas de salud y pérdida de competencias funcionales (problemas de discapacidad). Éste está agravado por la ausencia de seguro de salud y de diagnósticos médicos, problemas de automedicación y polifarmacia, así como consumo de alcohol, lo que limita su capacidad de adaptación para enfrentar las altas presiones del ambiente físico y socio-laboral. A su vez, en la ciudad el problema de salud se vincula a posibles deficiencias en el sistema sanitario para atender a las personas mayores más vulnerables, al no facilitar condiciones para el diagnóstico y mecanismos para la prevención de riesgos, como depresión, polifarmacia y alcohol (Nance, 2010).

Entre los estresores ambientales que determinan en mayor medida el riesgo de síntomas depresivos en trabajadores informales están los factores físicos y sociales del ambiente laboral. Los trabajadores en la vía pública, como pepenadores, están más expuestos a altas presiones ambientales, al desplazar 
pesadas cargas (cartones, chatarra) recorriendo amplias distancias y enfrentando potenciales peligros, como inclemencias climáticas, caídas por barreras arquitectónicas, atropellos e inseguridad, lo que tiene repercusiones negativas para su salud física y psicológica (García-Váldez et al., 2019). Aquellos otros que laboran en centros comerciales y pequeños comercios perciben problemas del ambiente físico-laboral (ruido y deficiente iluminación), aunque tienen menos riesgo de caídas por barreras arquitectónicas; y se enfrentan a problemas del ambiente socio-laboral, como falta de apoyo y discriminación, aunque observan menos riesgo de violencia, inseguridad y sobrecarga laboral. De todo ello se desprende que el riesgo de sintomatología depresiva se incrementa en ambientes laborales adversos, es decir, cuando se produce un desequilibro entre las capacidades de adaptación de los sujetos de avanzada edad y las presiones del ambiente (Lawton y Nahemow, 1973). Justamente, en el espacio público (calles, plazas), sobre todo, de ciudades en desarrollo, las personas mayores están más expuestas a peligros de caídas y atropellos (Nyman et al., 2013), así como a padecer aislamiento social, depresión y ansiedad (Vallée et al., 2011; Phillips et al., 2013; Rantakokko et al., 2014) por vivir en situación de inseguridad.

A diferencia de algunos estudios que indican que una ocupación laboral contribuye a mejorar el estado físico y psicológico durante el envejecimiento (Mortean et al., 2015), otras investigaciones concluyeron que determinados trabajos, sobre todo informales, podían tener efectos negativos sobre la salud y el bienestar de las personas mayores empleadas (Argüelles, 2014). Precisamente, los datos indican una elevada tasa de accidentes laborales (12,9\%) entre los adultos mayores del sector informal en Ciudad Juárez, muy superior al $2 \%$ que registraron las fuentes oficiales para la población total activa (IMSS, 2014). El riesgo de accidentes laborales entre personas con síntomas depresivos aumenta entre trabajadores de 75 y más años, sin estudios, mala salud, problemas físicos-sociales en el ambiente y deseo de cambiar de trabajo. A ello contribuyen también los fallos importantes en las inspecciones de trabajo y en los programas de empleo, consistentes en estrategias de incorporación al mercado de trabajo (Sánchez-González y Egea, 2011), así como refleja deficiencias en los registros de urgencias hospitalarias y en los protocolos de los programas de salud pública para diagnosticar y atender a personas mayores en riesgo de sintomatología depresiva y en situación de vulnerabilidad.
Los resultados indican que los síntomas depresivos variaban de manera significativa según lugar de trabajo, siendo los comercios informales y vía pública los que presentan más diferencias con el resto de lugares de trabajo. Al respecto, los factores que los explican varían según la tipología de trabajadores predominante en cada lugar de trabajo. Por ejemplo, entre los empleados informales de los comercios informales y vía pública, como pepenadores, se observaron mayores problemas de exclusión social, como precariedad económica y consumo de alcohol, asociadas con la sintomatología depresiva. Asimismo, se señala que los problemas del ambiente socio-laboral preocupan, sobre todo, a trabajadores de pequeños comercios y comercios ambulantes, mientras los del ambiente físico-laboral ocupan un lugar secundario entre sus prioridades.

En México el escenario de alta inseguridad ciudadana condiciona la vida cotidiana de sus habitantes, máxime de las personas mayores. Este hecho ha determinado el sesgo en el tamaño de la muestra, posiblemente, de aquellos colectivos más vulnerables y en empleos informales más expuestos a peligros laborales, como accidentes y violencia. Cabe la hipótesis de que en este grupo de edad exista una subestimación de la tasa de siniestralidad laboral, abuso y violencia en el trabajo. Por ello, es necesario realizar mayores esfuerzos gubernamentales en materia de coordinación entre inspección de trabajo y salud pública para detectar a las personas mayores en situación de riesgo. Asimismo, en futuras investigaciones sería recomendable disponer del historial médico de los participantes de edad y de sus familiares, con objeto de poder establecer mejores diagnósticos y posibilitar el diseño de estrategias de intervención en personas de edad en activo con la sintomatología depresiva.

En este trabajo los resultados revelan las conexiones entre el riesgo de síntomas depresivos, la vulnerabilidad social, y las condiciones laborales y ambientales. Sin embargo, al igual que otros estudios (Chen et al., 2009), se observa una complejidad metodológica para posibilitar la evaluación de la multiplicidad de factores que determinan la sintomatología depresiva, sobre todo, en trabajadores informales de avanzada edad. Una solución posible derivaría del uso de estudios longitudinales más concluyentes, basados en nuevos instrumentos metodológicos para medir la relación entre el riesgo de síntomas depresivos y los factores ambientales. Asimismo, es muy importante poner el foco en las condiciones específicas del trabajo informal en el envejecimiento en regiones en desarrollo, 
donde las personas mayores vulnerables se ven forzadas a continuar en el mercado de trabajo, incluso, en condiciones precarias y a costa de poner en riesgo su salud y calidad de vida.

En el mundo la caída de la fecundidad y el aumento de la esperanza de vida se asocian con el envejecimiento demográfico y la necesidad de prolongar la vida laboral para hacer sostenible el sistema público (pensiones, salud). Esta nueva realidad debe impulsar estrategias para favorecer el trabajo sostenible a lo largo de la vida laboral y, especialmente, a medida que aumenta la edad del trabajador, mediante la formación continua, la compatibilidad de la vida laboral y familiar, y la promoción del envejecimiento activo y saludable (Clas-Håkan, 2020).

Con objeto de mejorar el diagnóstico y la prevención de la sintomatología depresiva entre trabajadores informales de edad avanzada, es necesario favorecer la revisión de los programas de salud pública y de empleo encaminados a prevenir amenazas para su salud e integridad. También, las reformas laborales

\section{REFERENCIAS}

Aguilar-Navarro, S.G., y Ávila-Funes, J.A. (2007). La depresión: particularidades clínicas y consecuencias en el adulto mayor. Gaceta Médica de México, 143(2), pp. 141-148.

Aguilar-Navarro, S.G., Fuentes-Cantú, A., Ávila-Funes, J.A., y García-Mayo, E.J. (2007). Validez y confiabilidad del cuestionario del ENASEM para la depresión en adultos mayores. Salud Pública de México, 49(4), pp. 256-262.

Álvarez, C.V., Potes, M.P.E., y Merchán, M.E.P. (2016). Calidad de vida y trabajo informal en personas mayores en una ciudad intermedia colombiana, 2012-2013. Revista Brasileira de Geriatria e Gerontologia, 19(3), pp. 415-427. Doi:10.1590/180998232016019.140215 .

Argüelles, J.M. (2014). The Meaning of Work for Elderly Grocery Baggers in Monterrey, Mexico: A Precarious Job or a Social Opportunity?. The International Journal of Aging \& Society, 3(2), pp. 7-18. Doi:10.18848/2160-1909/CGP/v03i02/35238.

Ávila-Funes, J.A., Melano-Carranza, E., Payette, H., y Amieva, H. (2007). Síntomas depresivos como factor de riesgo de dependencia en adultos mayores. Salud Pública de México, 49(5), pp. 367375. deben promover la adaptación de las condiciones del entorno laboral para reducir la vulnerabilidad de las personas mayores trabajadoras ante los efectos del cambio climático (Chávez-Alvarado y Sánchez-González, 2016). Igualmente, se subraya la importancia de impulsar estudios para evaluar las políticas públicas para personas mayores en la región, así como diseñar y planificar ambientes laborales saludables y amigables, encaminados a propiciar la incorporación y participación de la creciente población activa envejecida.

Ciudad Juárez, y, en general, América Latina, deben preparase para enfrentar adecuadamente el reto de la depresión en los adultos mayores, a través de propiciar las condiciones sociales, de salud, laborales y ambientales adecuadas para impulsar el envejecimiento activo y saludable (Sánchez-González y Rodríguez, 2016). Precisamente, esta circunstancia obligará a diseñar ambientes de trabajo proactivos y amigables con las personas de edad, encaminados a favorecer su participación y empoderamiento, así como a enfrentar y prevenir la creciente amenaza de la depresión a nivel global.

Bauman, Z. (2009). La posmodernidad y sus descontentos. Madrid: Akal.

Bech, U. (2002). La sociedad del riesgo global. Madrid: Siglo XXI.

Cabañero-Martínez, M.J., Cabrero-García, J., Richart-Martínez, M., Muñoz, C.L., y Reig-Ferrer, A. (2007). Revisión estructurada de las escalas de depresión en personas mayores. International Journal of Clinical and Health Psychology, 7(3), pp. 823-846.

CCSPJP (2014). Las 50 ciudades más peligrosas del mundo 2014. México: Consejo Ciudadano para la Seguridad Pública y la Justicia Penal.

Chávez-Alvarado, R., y Sánchez-González, D. (2016). Envejecimiento vulnerable en hogares inundables y su adaptación al cambio climático en ciudades de América Latina: El caso de Monterrey. Papeles de Población, 22(90), pp. 9-42. Doi:10.22185/244 87147.2016.90.033.

Chen, W.Q., Siu, O.L., Lu, J.F., Cooper, C.L., y Phillips, D.R. (2009).Work stress and depression: The direct and moderating effects of informal social support and coping. Stress and Health, 25(5), pp. 431-443. Doi:10.1002/smi.1263. 
Clas-Håkan, N. (2020). Sustainable Work Ability and Aging. Basel, Switzerland: MDPI.

Conwell, Y., Van Orden, K., y Caine, E.D. (2012). Suicidio en ancianos. Psiquiatría Biológica, 19(4), pp. 99-142. Doi:10.1016/j.psiq.2012.09.003.

Dechent, C. (2008). Depresión geriátrica y trastornos cognitivos. Revista Hospital Clínico Universidad de Chile, 19, pp. 339-346.

Dunlop, D.D., Manheim, L.M., Song, J., Lyons, J.S., y Chang, R.W. (2005). Incidence of disability among preretirement adults: the impact of depression. American Journal of Public Health, 95(11), pp. 2003-2008. Doi:10.2105/AJPH.2004.050948.

Escobar, S. (2012). Los adultos mayores en el mundo del trabajo urbano. La Paz: HelpAge International.

Gallo, J.J., y Rabins, P.V. (1999). Depression without sadness: alternative presentations of depression in late life. American Family Physician, 60(3), pp. 820-826.

García-Sánchez, M.R., Reyes, J., Antúnez, T., Godínez, G., y Silva, A. (2016). Reforma laboral en México, ¿Solución a la justicia social? Revista Iberoamericana de las Ciencias Sociales y Humanísticas, 5(9), pp. 275-291.

García-Machín, E. (2006). Necesidad de la permanencia en el trabajo de los mayores de 60 años. Revista Cubana de Salud y Trabajo, 7(1-2), pp. 57-62.

García-Valdez, M.T., Sánchez-González, D., y RománPérez, R. (2019). Envejecimiento y estrategias de adaptación a los entornos urbanos desde la Gerontología Ambiental. Estudios Demográficos y Urbanos, 34(100), pp. 101-128. Doi:10.24201/ edu.v34i1.1810

Geib, L.T.C. (2012). Social determinants of health in the elderly. Ciência \& Saúde Coletiva, 17(1), pp. 123133. Doi:10.1590/S1413-81232012000100015.

Giatti, L., y Barreto, S.M. (2003). Saúde, trabalho e envelhecimento no Brasil. Cadernos de Saúde Pública, 19(3), pp. 759-771. Doi:10.1590/S0102$311 \times 2003000300008$.

Heikkinen,R.L., y Kauppinen, M. (2004). Depressive symptoms in late life: A 10-year follow-up. Archives of Gerontology and Geriatrics, 38(3), pp. 239-250. Doi:10.1016/j.archger.2003.10.004.

Hernández-Laos, E. (2013). Legislación laboral, sector informal y productividad multifactorial en México. Economía UNAM, 10(28), pp. 5-52.
IMSS (2014). Memorias estadísticas. México: Instituto Mexicano del Seguro Social.

INEGI (2015). Condiciones Socioeconómicas 2015. Aguascalientes: Instituto Nacional de Estadística y Geografía.

INEGI (2019). Encuesta Nacional de Ocupación y Empleo 2019. Aguascalientes: Instituto Nacional de Estadística y Geografía.

Karasek, R., y Theorell, T. (1990). Healthy work: Stress, productivity and the reconstruction of the working life. New York: Basic Books.

Lavretsky, H., Sajatovic, M., y Reynolds, C. (2013). Latelife Mood Disorders. Oxford: Oxford University.

Lawton, M.P., y Nahemow, L. (1973). Ecology and the aging process. In C. Eisdorfer y M.P. Lawton (Eds.), The psychology of adult development and aging (pp. 619-674). Washington DC.: American Psychological Association.

Lupien, S.J., McEwen, B.S., Gunnar, M.R., y Heim, C. (2009). Effects of stress throughout the lifespan on the brain, behaviour and cognition. Nature Reviews Neuroscience, 10(6), pp. 434-445. Doi: $10.1038 / \mathrm{nrn} 2639$.

Lyness, J.M., Duberstein, P.R., King, D.A., Cox, C., y Caine, E.D. (1998). Medical illness burden, trait neuroticism, and depression in older primary care patients. The American Journal of Psychiatry, 155(7), pp. 969-971. Doi:10.1176/ajp.155.7.969.

Martínez, J.M., Martínez, V.A., Esquivel, C.G., y Velasco, V.M. (2007). Prevalencia de depresión y factores de riesgo en el adulto mayor hospitalizado. Revista Médica del Instituto Mexicano del Seguro Social, 45(1), pp. 21-28.

Martínez de la Iglesia, J., Onís, M.C., Dueñas, R., Albert, C., Aguado, C., y Luque, R. (2002). Versión española del cuestionario de Yesavage abreviado (GDS) para el despistaje de depresión en mayores de 65 años: adaptación y validación. Medifam, 12(10), pp. 26-40.

Medina-Gómez, O., y López-Arellano, O. (2019). Informalidad laboral y derecho a la salud en México, un análisis crítico. Ciência \& Saúde Coletiva, 24(7), pp. 2583-2592. Doi:10.1590/141381232018247.14342017.

Melguizo, E., Acosta, A., y Castellano, B. (2012). Factores asociados a la calidad de vida de adultos mayores, Cartagena (Colombia). Salud Uninorte, 28(2), pp. 251-263. 
Millán, B. (2010). Factores asociados a la participación laboral de los adultos mayores mexiquenses. $\mathrm{Pa}$ peles de Población, 16(64), pp. 93-121.

Montoya, B.J., y Montes-de-Oca, H. (2009). Situación laboral de la Población adulta mayor en el Estado de México. Papeles de Población, 15(59), pp. 193-238.

Mortean, L., Schneider, I.J.C., Confortin, S.C., y Orsi, E. (2015). Paid Work and Physical Activity Preserve Functional Capacity in Elderly People. Gerontology and Geriatric Medicine, 2015(1), pp. 1-8. Doi:10.1177/2333721415608022.

Muñoz, O., García, C., y Durán, L. (Eds.) (2004). La saIud del adulto mayor: temas y debates, depresión en el anciano: una perspectiva general. México: Instituto del Seguro Social.

Nance, D.C. (2010). Depresión en el adulto mayor. En L.M. Gutiérrez (Ed.), Envejecimiento humano. Una visión transdisciplinaria (pp. 239-248). México: Instituto de Geriatría.

Nyman, S.R., Ballinger, C., Phillips, J.E., y Newton, R. (2013). Characteristics of outdoor falls among older people: a qualitative study. BMC Geriatrics, 13, pp. 125. Doi:10.1186/1471-2318-13-125.

OCDE (2016). Estudio de la OCDE sobre los sistemas de pensiones: México. México: Organización para la Cooperación y el Desarrollo Económicos.

Paz, J.A. (2010). Envejecimiento y Empleo en América Latina y el Caribe. Ginebra: Organización Internacional del Trabajo.

Peiró, J.M. (1999). Desencadenantes del estrés laboral. Madrid: Pirámide.

Phillips, D.R., Siu, O.L., Yeh, A.G., y Cheng, K.H. (2008). Informal social support and older persons' psychological well-being in Hong Kong. Journal of Cross-Cultural Gerontology, 23(1), pp. 39-55. Doi:10.1007/s10823-007-9056-0.

Phillips, J., Walford, N., Hockey, A., Foreman, N., y Lewis, M. (2013). Older people and outdoor environments: Pedestrian anxieties and barriers in the use of familiar and unfamiliar spaces. Geoforum, 47, pp. 113-124. Doi:10.1016/j.geoforum.2013.04.002.

Polanyi, K. (1989). La gran transformación. Critica del liberalismo económico. Madrid: Ediciones de La Piqueta.

Quintana, L., y Garza, B.E. 2017. La reforma laboral en México y sus efectos económicos. Revista do Tribunal Superior do Trabalho, 83(3), pp. 160-177.
Ramos, M. (2017). Envejecer siendo mujer, dificultades, oportunidades y retos. Barcelona: Bellaterra.

Rantakokko, M., Iwarsson, S., Vahaluoto, S., Portegijs, E., Viljanen, A., y Rantanen, T. (2014). Perceived environmental barriers to outdoor mobility and feelings of loneliness among community-dwelling older people. The Journals of Gerontology Series A: Biological Sciences and Medical Sciences, 69(12), pp. 1562-1568. Doi:10.1093/gerona/ glu069.

Sala, G.A. (2013). Empleo y desempleo entre los adultos mayores de la Ciudad Autónoma de Buenos Aires. Población de Buenos Aires, 10(17), pp. 7-26.

Sánchez-González, D. (2009). Contexto ambiental y experiencia espacial de envejecer en el lugar: el caso de Granada. Papeles de Población, 15(60), pp. $175-213$

Sánchez-González, D. (2015). Ambiente físico-social y envejecimiento de la población desde la gerontología ambiental y geografía: Implicaciones socioespaciales en América Latina. Revista de Geografía Norte Grande, 60, pp. 97-114. Doi:10.4067/ S0718-34022015000100006.

Sánchez-González, D., y Egea, C. (2011). Enfoque de vulnerabilidad social para investigar las desventajas socioambientales. Su aplicación en el estudio de los adultos mayores. Papeles de Población, 17(69), pp. 151-185.

Sánchez-González, D., y Rodríguez-Rodríguez, V. (Eds.) (2016). Environmental Gerontology in Europe and Latin America. Policies and perspectives on environment and aging. New York: Springer. Doi:10.1007/978-3-319-21419-1.

Sarró, M., Ferrer, A., Rando, Y., Formiga, F., y Rojas, S. (2013). Depression in the elderly: Prevalence and associated factors. Semergen, 39(7), pp. 354-360. Doi:10.1016/j.semerg.2013.01.007.

Seligman, M.E.P. (2008). Positive Health. Applied Pshychology, 57(1), pp. 3-18. Doi:10.1111/j.14640597.2008.00351.x.

Serra, M., y Germán, J.L. (2013). Polifarmacia en el adulto mayor. Revista Habanera de Ciencias Médicas, 12(1), pp. 142-151.

Spector, P.E. (1986). Perceived Control by Employees: A Meta-Analysis of Studies Concerning Autonomy and Participation at Work. Human Relations, 39(11), pp. 1005-1016. Doi:10.1177/001872678603901104. 
Tapia, M.S., Morales, J.J., Cruz, R.M., y De la Rosa, V. (2000). Depresión en el adulto mayor con enfermedad crónica. Revista de Enfermería del IMSS, 8(2), pp. 87-90.

Tuesca, R., Fierro, N., Molinares, A., Oviedo, F., PoloArjona, Y., Polo-Cueto, J., y Sierra, I. (2003). Los grupos de socialización como factor protector contra la depresión en personas ancianas: Baranquilla, Colombia. Revista Española de Salud Públi$c a, 77(5)$, pp. 595-604.

Tytherleigh, M.Y., Jacobs, P.A., Webb, C., Ricketts, C., y Cooper, C. (2007). Gender, Heath and Stress in English University Staff- Exposure or Vulnerability?. Applied Psychology, 56(2), pp. 267-287. Doi:10.1111/j.1464-0597.2006.00254.x.

Urbina, J.R., Flores, J.M., García, M.P., Torres, L., y Torrubias, R.M. (2007). Síntomas depresivos en personas mayores: Prevalencia y factores asociados. Gaceta Sanitaria, 21(1), pp. 37-42.

Vallée, J., Cadot, E., Roustit, C., Parizot, I., y Chauvin, P. (2011). The role of daily mobility in mental health inequalities: the interactive influence of activity space and neighbourhood of residence on depres- sion. Social Science \& Medicine, 73(8), pp. 11331144. Doi:10.1016/j.socscimed.2011.08.009.

Van-Gool, C.H., Kempen, G.I.J.M., Bosma, H., van Boxtel, M.P.J., Jolles, J., y van Eijk, J.T.M. (2007). Associations Between Lifestyle and Depressed Mood: Longitudinal Results from the Maastricht Aging Study. American Journal of Public Health, 97(5), pp. 887-894. Doi:10.2105/AJPH.2004.053199.

Verhaak, P.F.M., Dekker, J.H., de Waal, M.W.M., van Marwijk, H.W.J., y Comijs, H.C. (2014). Depression, disability and somatic diseases among elderly. Journal of Affective Disorders, 167(1), pp. 187-191. Doi:10.1016/j.jad.2014.05.057.

Vieco, G.F., y Abello, R. (2014). Factores psicosociales de origen laboral, estrés y morbilidad en el mundo. Psicología desde el Caribe, 31(2), pp. 354-385.

Von-Mühlenbrock-S, F., Gómez-D, R., González-V, M., Rojas-A, A., Vargas-G, L., y von-Mühlenbrock-P, C. (2011). Prevalencia de Depresión en pacientes mayores de 60 años hospitalizados en el Servicio de Medicina Interna del Hospital Militar de Santiago. Revista Chilena de Neuro-Psiquiatría, 49(4): 33133. Doi:10.4067/S0717-92272011000400004. 


\section{ANEXOS}

TABLA 1.

PERSONAS MAYORES SEGÚN SÍNTOMAS DEPRESIVOS, CARACTERÍSTICAS SOCIODEMOGRÁFICAS Y AMBIENTE LABORAL.

\begin{tabular}{|c|c|c|c|c|c|c|c|c|}
\hline \multirow[t]{2}{*}{ Variable } & \multicolumn{2}{|c|}{ Total } & \multirow{2}{*}{$\begin{array}{c}\begin{array}{c}\text { Ausencia de } \\
\text { depresión }\end{array} \\
(\%)^{(b)}\end{array}$} & \multirow{2}{*}{$\begin{array}{c}\begin{array}{c}\text { Síntomas } \\
\text { depresivos }\end{array} \\
(\%)^{(b)}\end{array}$} & \multirow{2}{*}{$\begin{array}{c}\text { Odds Ratio } \\
\text { (OR) }\end{array}$} & \multirow{2}{*}{ IC95\% } & \multirow{2}{*}{$\begin{array}{l}P \\
\text { (p) }\end{array}$} & \multirow{2}{*}{$\frac{\chi^{2}}{\text { (sig.) }}$} \\
\hline & (N) & $(\%)^{(\mathrm{a})}$ & & & & & & \\
\hline \multicolumn{9}{|l|}{ Sexo } \\
\hline Mujeres & 24 & 38,7 & 41,7 & 58,3 & 3,033 & $(1,05-8,77)$ & & 0,038 \\
\hline Varones & 38 & 61,3 & 68,4 & 31,6 & & & & \\
\hline Edad & & & & & & & $0,350 * *$ & \\
\hline 75> años & 24 & 38,7 & 54,2 & 45,8 & 1,297 & $(0,46-3,65)$ & & \\
\hline $65-74$ años & 38 & 61,3 & 60,5 & 39,5 & & & & \\
\hline Forma de convivencia & & & & & & & & 0,002 \\
\hline Vive solo & 18 & 29 & 66,7 & 33,3 & 2,643 & $(0,74-9,41)$ & & \\
\hline Cónyuge e hijos & 43 & 69,4 & 55,8 & 44,2 & & & & \\
\hline Otras personas & 1 & 1,6 & 100 & 0 & & & & \\
\hline Nivel educativo & & & & & & & $-0,297^{*}$ & \\
\hline Sin escolarizar & 9 & 14,5 & 66,7 & 33,3 & & & & \\
\hline Primaria incompleta & 25 & 40,3 & 40 & 60 & 3,545 & $(1,22-10,30)$ & & \\
\hline Primaria terminada & 18 & 29 & 55,6 & 44,4 & & & & \\
\hline Secundaria y estudios superiores & 10 & 16,2 & 100 & 0 & & & & \\
\hline Percepción de la salud & & & & & & & $-0,577^{* *}$ & \\
\hline Muy mala y mala salud & 8 & 12,9 & 25 & 75 & 5,1 & $(3,37-27,72)$ & & \\
\hline Regular de salud & 29 & 46,8 & 44,8 & 55,2 & & & & \\
\hline Buena y muy salud & 25 & 40,3 & 84 & 16 & & & & \\
\hline \multicolumn{9}{|l|}{ Discapacidad } \\
\hline Discapacidad física (motora) & 7 & 11,3 & 42,9 & 57,1 & 6,815 & $(1,30-35,79)$ & & \\
\hline Discapacidad visual & 9 & 14,5 & 55,6 & 44,4 & & & & \\
\hline Discapacidad auditiva & 3 & 4,8 & 0 & 100 & 2,565 & $(1,86-3,53)$ & $0,253^{*}$ & \\
\hline \multicolumn{9}{|l|}{ Consumo de medicamentos y alcohol(c) } \\
\hline Consumo $\geq 3$ medicamentos (polifarmacia) & 30 & 48,4 & 43,3 & 56,7 & 3,342 & $(1,16-9,61)$ & $0,532 * *$ & \\
\hline Consumo de alcohol & 15 & 12,9 & 46,7 & 53,3 & 1,841 & $(1,58-5,95)$ & $0,357^{* *}$ & \\
\hline \multicolumn{9}{|l|}{ Lugar de trabajo } \\
\hline Centro comercial & 31 & 50 & 51,6 & 48,4 & 1,705 & $(0,62-4,72)$ & & \\
\hline Pequeño comercio y taller & 15 & 24,2 & 86,7 & 13,3 & 0,147 & $(0,03-0,73)$ & & 0,023 \\
\hline Comercio ambulante y vía pública & 16 & 25,8 & 43,8 & 56,2 & 2,193 & $(1,69-6,96)$ & & 0,041 \\
\hline Factores del ambiente físico-laboral $^{(c)}$ & & & & & & & $0,284^{*}$ & \\
\hline Contaminación acústica (ruido) & 3 & 4,8 & 66,7 & 33,3 & 2,917 & $(0,25-34,00)$ & & \\
\hline Deficiente iluminación & 3 & 4,8 & 0 & 100 & 2,565 & $(1,86-3,53)$ & $0,265^{*}$ & \\
\hline Condiciones climáticas extremas & 2 & 3,2 & 0 & 100 & 2,5 & $(1,83-3,41)$ & $0,354^{* *}$ & \\
\hline Barreras arquitectónicas (caídas) & 6 & 9,7 & 66,7 & 33,3 & 0,667 & $(0,11-3,94)$ & & \\
\hline Tráfico y riesgo de atropellos & 4 & 6,5 & 50 & 50 & 1,417 & $(0,19-10,77)$ & & \\
\hline Distancia & 3 & 4,8 & 0 & 100 & 2,565 & $(1,86-3,53)$ & $0,253^{*}$ & \\
\hline Factores del ambiente social-laboral(c) & & & & & & & $0,370^{* *}$ & \\
\hline Falta de apoyo & 12 & 19,4 & 50 & 50 & 1,5 & $(0,42-5,31)$ & & \\
\hline Violencia/abuso (extorción) & 32 & 51,6 & 53,1 & 46,9 & 1,524 & $(0,55-4,21)$ & $0,261^{*}$ & \\
\hline Malas relaciones sociales & 21 & 33,9 & 42,9 & 57,1 & 2,571 & $(1,25-7,56)$ & $0,301 *$ & \\
\hline Discriminación (decisiones, promociones) & 4 & 6,5 & 0 & 100 & 2,636 & $(1,90-3,67)$ & $0,309^{*}$ & \\
\hline Sobrecarga laboral & 25 & 40,3 & 48 & 52 & 2 & $(1,24-5,63)$ & $0,304 *$ & \\
\hline Inseguridad laboral & 20 & 32,3 & 50 & 50 & 1,625 & $(0,55-4,76)$ & $0,323^{*}$ & \\
\hline Bajos salarios & 56 & 90,3 & 55,4 & 44,6 & 4,032 & $(0,44-36,78)$ & & \\
\hline \multicolumn{9}{|l|}{ Accidentes laborales (últimos 6 meses) (c) } \\
\hline Accidentes laborales (últimos 6 meses) & 8 & 12,9 & 75 & 25 & 0,417 & $(0,07-2,25)$ & & 0,024 \\
\hline \multicolumn{9}{|l|}{ Deseo de cambiar de trabajo ${ }^{(c)}$} \\
\hline Deseo de cambiar de trabajo & 24 & 38,7 & 58,3 & 41,7 & 0,982 & $(0,35-2,77)$ & 0,323 & \\
\hline Total & 62 & 100 & 58,1 & 41,9 & & & & \\
\hline
\end{tabular}

(a) Porcentaje por columnas; (b) Porcentaje por filas; (c)Porcentajes calculados sobre el total de casos a partir de pregunta multirrespuesta.

$(* *)$ Correlación significativa al nivel 0,01 (bilateral); $\left({ }^{*}\right)$ Correlación significativa al nivel 0,05 (bilateral).

$\left(x^{2}\right)$ Prueba de chi-cuadrado de Pearson (Sig. asintótica bilateral).Fuente y elaboración propia 
TABLA 2.

MODELO DE REGRESIÓN LOGÍSTICA ENTRE SINTOMATOLOGÍA DEPRESIVA Y VARIABLES SOCIODEMOGRÁFICAS Y AMBIENTALES.

\begin{tabular}{|l|c|c|}
\hline \multicolumn{1}{|c|}{ Dimensión } & Variable & Coeficientes tipificados beta \\
\hline Sociodemográfica & Sin estudios terminados & 0,017 \\
Salud & Mala salud percibida & 0,003 \\
\hline
\end{tabular}

$\mathrm{N}=62$. Porcentaje pronosticado por el modelo $=75,8 \% . \mathrm{R}^{2}$ de Nagelkerke $=0,309$. Método por pasos hacia delante (Wald).

Paso 10 Edad (75 y más años), Estudios (sin estudios terminados), Salud (Mala salud percibida), Polifarmacia,

Problemas del ambiente físico-laboral, Problemas del ambiente socio-laboral, y Accidentes laborales.

Fuente y elaboración propia

TABLA 3.

MODELO DE REGRESIÓN LOGÍSTICA ENTRE SINTOMATOLOGÍA DEPRESIVA Y FACTORES DEL AMBIENTAL LABORAL INFORMAL.

\begin{tabular}{|l|c|c|c|}
\hline \multicolumn{1}{|c|}{ Dimensión } & Variable & Coeficientes tipificados beta & Odds ratio \\
\hline Ambiente físico-laboral & Distancias & 0,049 & 0,064 \\
& Condiciones climáticas extremas & 0,999 \\
Ambiente socio-laboral & Mala salud percibida & 0,016 & 0,156 \\
\hline
\end{tabular}

$\mathrm{N}=62$. Porcentaje pronosticado por el modelo $=83,9 \%$. R2 de Nagelkerke $=0,365$. Método por pasos hacia delante (Wald).

Paso 1 - Barreras arquitectónicas, Distancia, Condiciones climáticas extremas, Malas relaciones sociales, Sobrecarga laboral, e Inseguridad laboral.

Fuente y elaboración propia.

TABLA 4.

ANOVA DE UN FACTOR. SÍNTOMAS DEPRESIVOS SEGÚN LUGAR DE TRABAJO

\begin{tabular}{|l|c|c|c|c|c|}
\hline \multicolumn{3}{|c|}{ Síntomas depresivos } \\
\hline & Suma de cuadrados & gl & Media cuadrática & F & Sig. \\
\hline Inter-grupos & 1,684 & 2 & 0,842 & 3,704 & 0,031 \\
Intra-grupos & 13,413 & 59 & 0,227 & \\
Total & 15,097 & 61 & & \\
\hline
\end{tabular}

Fuente y elaboración propia.

TABLA 5.

PRUEBA POST HOC DE LA VARIANZA. TEST BONFERRONI. SÍNTOMAS DEPRESIVOS SEGÚN LUGAR DE TRABAJO

\begin{tabular}{|c|c|c|c|c|c|c|}
\hline \multirow[b]{2}{*}{ (I) Lugar de trabajo } & \multirow[b]{2}{*}{ (J) Lugar de trabajo } & \multirow{2}{*}{$\begin{array}{c}\text { Diferencia de } \\
\text { medias (I-J) }\end{array}$} & \multirow[b]{2}{*}{ Error típico } & \multirow[b]{2}{*}{ Sig. } & \multicolumn{2}{|c|}{ Intervalo de confianza al $95 \%$} \\
\hline & & & & & $\begin{array}{l}\text { Límite } \\
\text { inferior }\end{array}$ & $\begin{array}{l}\text { Límite } \\
\text { superior }\end{array}$ \\
\hline \multirow[t]{2}{*}{ Centro comercial } & Pequeño comercio y taller & $-0,351$ & 0,15 & 0,068 & $-0,72$ & 0,02 \\
\hline & Comercio ambulante y vía pública & $0,479 *$ & 0,147 & 0,045 & 0,28 & 0,44 \\
\hline \multirow[t]{2}{*}{ Pequeño comercio y taller } & Centro comercial & 0,351 & 0,15 & 0,068 & $-0,02$ & 0,72 \\
\hline & Comercio ambulante y vía pública & $0,429 *$ & 0,171 & 0,028 & 0,01 & 0,85 \\
\hline \multirow[t]{2}{*}{ Comercio ambulante y vía pública } & Centro comercial & $-0,479 *$ & 0,147 & 0,045 & $-0,44$ & $-0,28$ \\
\hline & Pequeño comercio y taller & $-0,429 *$ & 0,171 & 0,028 & $-0,85$ & $-0,01$ \\
\hline
\end{tabular}

$\left({ }^{*}\right)$ La diferencia de medias es significativa al nivel 0.05 .

Fuente y elaboración propia. 
TABLA 6.

PERSONAS MAYORES CON SÍNTOMAS DEPRESIVOS SEGÚN LUGAR DE TRABAJO Y FACTORES AMBIENTALES. CORRELACIONES.

\begin{tabular}{|c|c|c|c|}
\hline \multirow{4}{*}{ Variables } & \multicolumn{3}{|c|}{ Síntomas depresivos } \\
\hline & \multicolumn{3}{|c|}{ Lugar de trabajo } \\
\hline & Centros comerciales & Pequeños comercios y taller & $\begin{array}{c}\text { Comercios ambulantes y vía } \\
\text { pública }\end{array}$ \\
\hline & (p) & (p) & (p) \\
\hline Nivel educativo & & $-0,569 *$ & \\
\hline Dificultad económica & & $0,517^{*}$ & $0,663^{* *}$ \\
\hline Percepción de la salud & $-0,559 * *$ & $-0,701^{* *}$ & $-0,637 * *$ \\
\hline Grado de discapacidad & & $0,728 * *$ & \\
\hline Consumo $\geq 3$ medicamentos (polifarmacia) & $0,516 * *$ & & $0,600 * *$ \\
\hline Consumo de alcohol & & & $0,618^{* *}$ \\
\hline Factores del ambiente físico-laboral & & & \\
\hline Contaminación acústica (ruido) & & & \\
\hline Deficiente iluminación & & & \\
\hline Condiciones climáticas extremas & & & \\
\hline Barreras arquitectónicas (caídas) & & & \\
\hline Tráfico y riesgo de atropellos & & & \\
\hline Distancia & & & \\
\hline Factores del ambiente social-laboral & & $0,721^{* *}$ & $0,572^{*}$ \\
\hline Falta de apoyo & & & \\
\hline Violencia/abuso (extorción) & & $0,650 * *$ & $0,516 *$ \\
\hline Malas relaciones sociales & & $0,681 * *$ & $0,733 * *$ \\
\hline Discriminación (decisiones, promociones) & & & \\
\hline Sobrecarga laboral & & $0,650 * *$ & \\
\hline Inseguridad laboral & $0,372^{*}$ & $0,784^{* *}$ & \\
\hline Bajos salarios & & & \\
\hline Accidentes laborales (últimos 6 meses) & & & \\
\hline Deseo de cambiar de trabajo & $0,372^{*}$ & $0,650 * *$ & $0,516^{*}$ \\
\hline
\end{tabular}

(**) Correlación significativa al nivel 0,01 (bilateral);

$\left({ }^{*}\right)$ Correlación significativa al nivel 0,05 (bilateral).

Fuente y elaboración propia. 